\title{
Bordetella holmesii endocarditis in a patient with systemic lupus erythematous treated with immunosuppressive agents
}

Sir,

Bordetella holmesii, formerly designated as CDC non-oxidizer group 2, was recently classified as a member of the genus Bordetella. ${ }^{1}$

This pathogen has been reported as a rare cause of bactaeremia, respiratory tract infection, and endocarditis, particularly in younger patients. ${ }^{1,2-9}$ Anatomic or functional asplenia, renal transplantation, HIV infection, long-term steroid therapy, Hodgkin's lymphoma, and chronic obstructive pulmonary disease are all predisposing conditions for invasive disease. ${ }^{2-9}$ Bordetella spp. represents one of the non-HACEK (Haemophilus species, Actinobacillus actinomycetemcomitans, Cardiobacterium hominis, Eikenella corrodens, and Kingella species) Gram-negative rods that can cause endocarditis. ${ }^{6,9}$ Infective endocarditis (IE) caused by non-HACEK Gram-negative bacilli is a rare and poorly understood disease; only few cases have been described so far and all present a high mortality rate $(24 \%) .{ }^{10}$

From 1972 to 2011, only two cases of Bordetella holmesii endocarditis have been found in a review of the English literature. The diagnosis of B. holmesii IE was confirmed by the presence of a valvular vegetation in only one of these two cases, and was documented through the use of the echocardiography; in the other case, the diagnosis was only clinically suspected.

Here we report a case of IE caused by Bordetella holmesii in a patient affected by systemic lupus erythematous (SLE) and receiving an immunosuppressive treatment with azathioprine and prednisone.

On March 2011, a 48-year-old male patient was admitted to the Infectious Diseases Department of the San Martino University Hospital of Genoa, Italy, due to a long history of fever and fatigue. His medical history included an autoimmune thyroiditis and a recent diagnosis of SLE, which had been based on physical examination and laboratory test results (positivity of antinuclear antibody test).

Before this hospitalization, no organ involvement of SLE had been described and no new sign was diagnosed on this matter at the Infectious Diseases Department. In his medical history, the patient had received remittent courses of prednisone therapy $(25 \mathrm{mg} / \mathrm{die})$ for his rheumatic conditions. On 1 February 2011, due to a persistent febrile state, azathioprine was introduced to his therapeutic scheme. During this period, a positron emission tomography/computed tomography scan was performed and it documented no evidence of metabolically active disease. However, due to the presence of a diastolic murmur on auscultation, a transthoracic echocardiography (TTE) was performed, which documented the presence of mobile vegetation on a bicuspid aortic valve, with moderate-severe aortic regurgitation and left ventricle overload. No lesions compatible with Libman-Sacks endocarditis existed prior to this episode.

The immunosuppressive therapy was then discontinued and the patient was hospitalized at the Infectious Diseases Department with the diagnosis of suspected IE. His temperature was $36.3^{\circ} \mathrm{C}$, heart rate was $90 \mathrm{bpm}$, and blood pressure was $125 / 65 \mathrm{mmHg}$. Physical examination revealed normal $\mathrm{S} 1$ and $\mathrm{S} 2$ sounds, but a $2 / 6$ diastolic murmur on the aortic auscultation site was detected. Laboratory test results showed elevated C-reactive protein $(90.9 \mathrm{mg} / 1$, normal range: $0-5 \mathrm{mg} / \mathrm{l}$ ) and no leukocytosis. Serial sets of blood cultures were performed and colonies of Gramnegative rods grew from five of them. The isolated pathogen grew on blood and chocolate agar plates after 2 days of incubation, but showed a very limited growth on MacConkey agar. The strain produced a diffusible brown pigment on blood agar plate and was catalase negative. Initially, it was not possible to identify the isolate neither through routine laboratory procedure by using VITEK 2 AES (BioMérieux, Durham, NC, USA) nor by the API NE and NH strips (BioMérieux). The strain was subsequently identified as Bordetella holmesii by sequence analysis of the hypervariable regions V3 and V6 of the 16S rRNA gene; by insertion elements (IS1001, IS1002, and IS481) analysis; by using previously described primers and conditions, and by differential biochemical tests (oxidase, nitrate reduction, and urease production) used to differentiate Bordetella 
species. ${ }^{11-13}$ Antimicrobial susceptibility testing was performed by the disk diffusion method. Results were interpreted according both to the Clinical and Laboratory Standard Institute break-points for fastidious bacteria and break-points for members of the family of Enterobacteriaceae [amikacin susceptible (S), gentamicin (S), piperacillin (S), piperacillin/tazobactam (S), ceftazidime (S), cefepime (S), cefotaxime resistant $(\mathrm{R})$ and aztreonam $(\mathrm{R})$, imipenem $(\mathrm{S})]^{14,15}$

Once having diagnosed the IE, an empirical antimicrobial treatment with piperacillin/tazobactam (4.5 g IV q6h) and gentamicin (80 mg IV q8h) was started. On day 3, piperacillin/tazobactam was substituted by meropenem ( $1 \mathrm{~g}$ IV q8h) because antimicrobial susceptibility patterns indicated that the isolate (not yet identified) was probably an extendedspectrum beta-lactamases (ESBL)-producer strain, since all blood isolates were resistant to cefotaxime. After 7 days of antimicrobial treatment, because of the persistence of severe aortic regurgitation and the onset of left ventricle overload, the patient urgently underwent an aortic valve replacement with a mechanical valve (St Jude 23). After surgery and antibiotic therapy, C-reactive protein levels returned to normal and, after 4 weeks of treatment, antimicrobial therapy was discontinued. After the surgical procedure, a TTE was performed, and it showed no signs of paravalvular leaks, prosthesis dehiscence, or thrombosis. After 6 months of follow-up, the patient remained in good clinical conditions and without bacterial recurrences.

To our knowledge, this is the first reported case of Bordetella holmesii infection in a patient with SLE. Bordetella spp. are traditionally regarded as human respiratory tract colonizers. However, they have been described as causative agents of infections such as bactaeremia, pneumonia and, rarely, endocarditis. Overall, only two cases of endocarditis have been previously reported. The first patient, a 25-year-old man who presented a medical history of radiation therapy for stage IIA Hodgkin's lymphoma, had a bicuspid aortic valve and a TTE revealed the presence of a valve vegetation. The second patient, a previously healthy 14-year-old girl, had a thickened mitral valve discovered at TTE. 3,7

In the case presented, Bordetella holmesii IE developed in a 48-year-old patient with SLE, previously treated with azathioprine and prednisone, who recovered completely after surgery and 4 weeks of antibiotic treatment. On hospital admission, we decided to administer piperacillin-tazobactam because of the absence of fever, the absence of severe clinical conditions, and no reported prior antibiotic use. However, despite the absence of a clinical worsening, we considered the choice of a new highly bactericidal drug with a potent and rapid activity, because of the potential severity of the infection. We wanted to be cautious, thinking about the increasing incidence of infections caused by ESBLs producing bacteria and considering that the activity of piperacillin-tazobactam on ESBLs producers cannot be easily predicted in vitro. ${ }^{16}$ Therefore, we decided to substitute piperacillin/tazobactam with meropenem, even though the isolate was susceptible to piperacillin/tazobactam in vitro. As predicted, blood cultures performed after 3 days of piperacillin/tazobactam therapy were positive for Gram-negatives rods, while blood cultures performed after the introduction of meropenem were negative, supporting our choice. Moreover, the isolated rod, subsequently identified as Bordetella holmesii, was in vitro resistant to cefotaxime, suggesting the production of ESBLs.

In a previously published analysis of 26 strains of $B$. holmesii, MIC ranges of penicillins and third-generation cephalosporins were elevated (varying from 8 to $>16 \mathrm{mg} / \mathrm{l}$ for penicillin, from 1 to $8 \mathrm{mg} / \mathrm{l}$ for ampicillin, and from 4 to $>8 \mathrm{mg} / \mathrm{l}$ for cefotaxime), suggesting the possibility of a poor clinical response to these drugs. ${ }^{8}$ More data are needed to achieve an adequate interpretation of in vitro Bordetella holmesii susceptibility. Although B. holmesii has been isolated from cultures of US patients nasopharyngeal specimens, the source of Bordetella bactaeremia remains uncertain. ${ }^{17}$ Modes of acquisition also remain to be identified, as no person-to-person transmission has been described. ${ }^{9}$ Bordetella holmesii may act as an opportunistic pathogen in particular clinical conditions such as hyposplenism or asplenia, conditions that are also associated with SLE and infections with encapsulated pathogens. ${ }^{18}$ While the presence of a polysaccharide capsule in Bordetella holmesii strains remains unknown, other Bordetella spp. are likely to encode a capsule. ${ }^{19}$ Therefore, even if the paucity of available data does not allow a clear identification of predisposing factors for invasive infections by $B$. holmesii, we want to emphasize that, despite the small number of reports, underlining immunocompromising conditions, such as steroid treatment or haematological malignancies, hyposplenism, and the presence of a native bicuspid aortic valve may be all factors associated with the risk of Bordetella holmesii disease.

Matteo Bassetti, Elena Nicco, Daniele Roberto Giacobbe, Anna Marchese, Erika Coppo, Ramona Barbieri, Claudio Viscoli

Santa Maria Misericordia Hospital, Udine and San Martino Hospital, Genoa, Italy

\section{References}

1 Weyant RS, Hollis DG, Weaver RE, Amin MF, Steigerwalt AG, O'Connor SP, et al. Bordetella holmesii sp. nov., a new gram negative species associated with septicemia. J Clin Microbiol. 1995;33:1-7. 
2 Lindquist SW, Weber DJ, Mangum ME, Hollis DG, Jordan J. Bordetella holmesii sepsis in an asplenic adolescent. Pediatr Infect Dis J. 1995;14:813-5.

3 Tang Y-W, Hopkins MK, Kolbert CP, Hartley PA, Severance PJ, Persing DH. Bordetella holmesii-like organisms associated with septicemia, endocarditis, and respiratory failure. Clin Infect Dis. 1998;26:389-92.

4 Morris JT, Myers M. Bacteremia due to Bordetella holmesii. Clin Infect Dis. 1998;27:912e3.

5 Njamkepo E, Delisle F, Hagege I, Gerbaud G, Guiso N. Bordetella holmesii isolated from a patient with sickle cell anemia: analysis and comparison with other Bordetella holmesi isolates. Clin Microbiol Infect. 2000;6:131-6.

6 Greig JR, Gunda SS, Kwan JT. Bordetella holmesii bacteraemia in an individual on haemodialysis. Scand J Infect Dis. 2001;33:716-7.

7 Russell FM, Davis JM, Whipp MJ, Janssen PH, Ward PB, Vyas JR, et al. Severe Bordetella holmesii infection in a previously healthy adolescent confirmed by gene sequence analysis. Clin Infect Dis. 2001;33:129-30.

8 Shepard CW, Daneshvar MI, Kaiser RM, Ashford DA, Lonsway D, Patel JB, et al. Bordetella holmesii bacteremia: a newly recognized clinical entity among asplenic patients. Clin Infect Dis. 2004:38:799-804.

9 Panagopoulos MI, Saint Jean M, Brun D, Guiso N, Bekal S, Ovetchkine P, et al. Bordetella holmesii bacteremia in asplenic children: report of four cases initially misidentified as Acinetobacter lwoffii. J Clin Microbiol. 2010;48:3762-4.

10 Morpeth S, Murdoch D, Christopher H, Karchmer AW Pappas P, Levine D, et al. Non-HACEK Gram-negative bacillus endocarditis. Ann Intern Med. 2007;147:829-35.

11 Chakravorty S, Helb D, Burday M, Connel N, Alland D. A detailed analysis of $16 \mathrm{~S}$ ribosomia RNA gene segments for the diagnosis of pathogenic bacteria. J Microbiol Methods. 2007;69:330-9.

12 Roorda L, Buitenwerf J, Ossewaarde JM, Van der Zee A. A real-time PCR assay with improved specificity for detection and discrimination of all clinically relevant Bordetella species by the presence and distribution of three Insertion Sequence elements. BMC Res Notes. 2011;4:11.

13 Loeffelholz MJ, Sanden GN. Bordetella. In: Manual of clinical microbiology, 9th edition. Murray PR, Barona EJ, Jorgenseng JH, Landry ML, Pfaller MA, editors. Washington, DC: ASM Press; 2007. p. 803-14.

14 Cockerill FR. Methods for antimicrobial dilution and disk susceptibility testing of infrequently isolated or fastidious bacteria. M45-A2. Wayne, PA: Clinical and Laboratory Standards Institute (CLSI); 2010.

15 Cockerill FR. Performance standards for antimicrobial susceptibility testing: twenty-first informational supplement. M100S21. Wayne, PA: Clinical and Laboratory Standards Institute (CLSI); 2011.

16 Livermore DM. Determinants of the activity of $\beta$-lactamase inhibitor combinations. J Antimicrob Chemother. 1993;31 Suppl A:9-21.

17 Yih WK, Silva EA, Ida J, Harrington N, Lett SM, George H. Bordetella holmesii-like organisms isolated from Massachusetts patients with pertussis-like symptoms. Emerg Infect Dis. 1999;5:441-3.

18 Pines A, Kaplinsky N, Olchovsky D, Holtzman E, Frankl O. Hyposplenism in systemic lupus erythematosus. $\mathrm{Br} J$ Rheumatol. 1983;22:176-8.

19 Neo Y, Li R, Howe J, Hoo R, Pant A, Ho S, et al. Evidence for an intact polysaccharide capsule in Bordetella pertussis. Microbes Infect. 2010;12:238-45. 